Check for updates

Cite this: J. Anal. At. Spectrom., 2018, 33,1076

Received 8th January 2018

Accepted 12th March 2018

DOI: $10.1039 / c 8 j a 00006 a$

rsc.li/jaas

\section{Accurate measurement of uranium isotope ratios in solid samples by laser ablation multi-collector inductively coupled plasma mass spectrometry $\dagger$}

\begin{abstract}
Z. Varga, D* M. Krachler, A. Nicholl, M. Ernstberger, T. Wiss, M. Wallenius and K. Mayer
A multi-collector inductively coupled plasma mass spectrometer (MC-ICP-MS) coupled to a $213 \mathrm{~nm}$ nslaser was used to measure uranium isotope ratios $\left({ }^{234} \mathrm{U} /{ }^{238} \mathrm{U},{ }^{235} \mathrm{U} /{ }^{238} \mathrm{U}\right.$, and $\left.{ }^{236} \mathrm{U} /{ }^{238} \mathrm{U}\right)$ in six solid nuclear certified reference materials (CRMs). The powdered CRMs, containing depleted, natural and low enriched uranium $(U)$, were pressed into pellets, for straightforward handling of the nuclear material. Being analysed without dissolution, the experimental $U$ isotope ratios of the solid materials were in good agreement with the corresponding certified values. The measured $U$ isotope ratios in the samples were corrected for mass bias using a pelletised CRM containing nominally $2 \%$ enriched ${ }^{235} \mathrm{U}$. Scanning electron microscopy investigations of an actual sample from which material was previously ablated revealed that only $5 \mathrm{ng}$ of sample is consumed during a single measurement. As such laser ablation MCICP-MS can be regarded as quasi-non-destructive and therefore is an ideal technique for the rapid but still accurate and precise determination of the uranium isotopic composition in solid samples for nuclear safeguards and forensic purposes. The proposed analytical procedure essentially assists the characterisation of the illicit nuclear material for hazard and origin assessment. Moreover, as the sample is also an evidence, it can be analysed with further techniques.
\end{abstract}

\section{Introduction}

Uranium (U) isotopic composition is widely measured in nuclear materials ( $\mathrm{U}$ oxides, $\mathrm{U}$ nitrate or $\mathrm{UF}_{6}$ ) for process and quality control purposes. Uranium has been measured accurately and timely in the starting material, in any intermediate samples and in the end product of the fuel cycle to make up a material balance. This nuclear safeguards system led by the International Atomic Energy Agency was set up to avoid the diversion of nuclear material. ${ }^{1,2}$ Thus, if such material gets out of regulatory control (despite physical protection and nuclear safeguards) and is subsequently detected and confiscated, detailed analyses are required to assess the hazard, intended use and origin of the material. These measurements, often referred to as nuclear forensics, involve the comprehensive analysis of the material in question. ${ }^{3-5}$ Among the suite of parameters investigated (e.g. inorganic impurities, chemical composition, $\mathrm{Pu}, \mathrm{Pb}, \mathrm{Sr}$, and $\mathrm{Nd}$ isotopic composition, and production date), the $\mathrm{U}$ isotopic composition is primarily important, as it indicates the intended use and source of the material.

European Commission, Joint Research Centre, Directorate for Nuclear Safety and Security, P.O. Box 2340, 76125 Karlsruhe, Germany. E-mail: Zsolt.Varga@ec. europa.eu

$\dagger$ Electronic supplementary information (ESI) available: MC-ICP-MS measurement conditions. See DOI: 10.1039/c8ja00006a
In order to ensure that the produced material has the desired isotopic composition or to confirm the material balance in safeguards, the isotope ratios have to be measured accurately and precisely. Moreover, for illicit material these values usually have to be sufficiently precise to identify its origin by comparing the experimental results with known values in databases. Several analytical techniques are applied routinely to determine $\mathrm{U}$ isotope ratios, such as traditional radioanalytical techniques, e.g. gamma spectrometry and alpha spectrometry, or mass spectrometric methods, such as thermal ionisation mass spectrometry (TIMS) ${ }^{6}$ and inductively coupled plasma mass spectrometry (ICP-MS).$^{7-9}$ ICP-MS instruments with several detectors (multi-collector ICP-MS and MC-ICP-MS) offer high precision due to the simultaneous measurement of the relevant isotopes.

The above mentioned analytical techniques typically require a liquid sample for analysis. The major drawback of such destructive techniques is the fact that they are normally timeconsuming and generate radioactive waste, a feature that is critical when dealing with nuclear material. Furthermore, even though for the actual measurement only a small amount of material is needed (less than $1 \mathrm{mg}$ ), a substantially higher quantity needs to be dissolved to ensure a representative sample. Coupling of laser ablation (LA) with ICP-MS overcomes these issues, as it uses a laser beam to ablate a minute portion of the material of interest and transfers it to the ICP-MS. Solid samples can be analysed directly without any sample preparation, and therefore the amount of nuclear waste is reduced 
significantly. Furthermore, the LA-based measurement is faster compared to the more laborious classical solution-based analysis. Results from LA-MC-ICP-MS are typically available within a few hours as time consuming steps such as weighing, dissolution or chemical separation of the sample in question are avoided.

LA uses a high-energy laser beam, and thus the difficult-todissolve fractions of a material, e.g. refractory components, can also be measured in this way. Additionally, as only a small sub-sample from the surface is analysed, but on multiple individual spots, LA-ICP-MS analysis may reveal sample inhomogeneity and consequently provide hints on the processing history of the material as proposed by Kell and Jovanovic. ${ }^{10}$ LA-ICP-MS has been used to measure nuclear samples, such as hot particles, ${ }^{\mathbf{1 1}, \mathbf{1 2}}$ irradiated nuclear fuels ${ }^{\mathbf{1 3}}$ or uranium ore concentrates. ${ }^{14}$ LA has also been applied to nuclear forensic samples, ${ }^{15,16}$ but only using single-collector instruments so far. While providing a powerful analytical tool, LA-ICP-MS is not yet used routinely for safeguards and nuclear forensics.

The present work aims at developing a fast but accurate and precise $U$ isotope ratio measurement method $\left({ }^{234} \mathrm{U} /{ }^{238} \mathrm{U}\right.$, ${ }^{235} \mathrm{U} /{ }^{238} \mathrm{U}$, and ${ }^{236} \mathrm{U} /{ }^{238} \mathrm{U}$ ) for solid nuclear samples employing LA-MC-ICP-MS. Certified nuclear reference materials (CRMs) of different ${ }^{235} \mathrm{U}$ enrichments ranging from depleted to lowenriched uranium were used to validate the experimental results. The CRMs are provided as powders, from which pressed pellets were prepared to (i) avoid the contamination of the LA cell and (ii) to mimic $U$ fuel pellets. The developed measurement procedure, the coupling of the LA system and the achieved accuracy and precision of $\mathrm{U}$ isotopic analysis are described in the paper.

\section{Materials and experimental methods}

The investigated specimens were $\mathrm{U}$ standard reference materials obtained as $\mathrm{U}_{3} \mathrm{O}_{8}$ from the National Bureau of Standards (NBS, Washington DC, USA) and presently distributed by the New Brunswick Laboratory (NBL, Argonne, IL, USA) as well as uranium ore concentrates (UOCs). ${ }^{17}$ The CRMs in their specified isotopic composition have originally been withdrawn from the cascade as the $\mathrm{UF}_{6}$ (gas) form, followed by using hydrolysis/ dissolution to prepare the CRMs. ${ }^{18}$ Thus they are considered homogeneous chemically even at the sub-micron level. All CRMs are certified for their $U$ isotopic composition. The analysed UOCs are industrial materials, and thus some degree of inhomogeneity is expected, even if they have been homogenised before distribution. The two UOC materials containing natural U have certified (IAEA-9449) or reported (IAEA-9064) values for their $\mathrm{U}$ isotopic composition.

For the reasons outlined above, the powder materials were pressed into pellets before the actual measurement. No binder was used for pellet preparation. The materials were pressed with an X-PRESS hydraulic laboratory press (Spex Industries, Metuchen, USA) applying a force of 2 tons for 8 minutes. The pellet diameter was $5 \mathrm{~mm}$, and the height was approximately 1 $\mathrm{mm}$. Pre-cleaned plastic covers were used on the dyes to exclude the contamination of the hydraulic press from the nuclear samples and to avoid cross-contamination. Before each measurement sequence the ICP-MS was optimised daily (torch position, gas flows, and voltages) using a $50 \mathrm{ng} \mathrm{g}^{-1}$ multielemental solution (Inorganic Ventures, Christiansburg, USA). The optimisation aimed at achieving the highest sensitivity and stability of the acquired U signal.

\section{Experimental set-up}

A NuPlasma ${ }^{\mathrm{TM}}$ (NU Instruments, Oxford, United Kingdom) double-focusing multi-collector inductively coupled plasma mass spectrometer (MC-ICP-MS), equipped with 11 Faraday detectors and 3 discrete dynode electrode multipliers, was used for the $\mathrm{U}$ isotope ratio measurements. The instrument was operated in low mass resolution mode $(R=300)$. The samples were introduced via a T-connection either through an Aridus II desolvation unit (CETAC Technologies Inc., Omaha, NE, USA) as a solution or through a NWR-213 laser ablation unit (ESI, Huntingdon, UK) as a solid sample. This particular set-up enables both the fast optimisation of the LA-MC-ICP-MS instrument and the rapid switching between the two sample introduction systems. The desolvating nebuliser was only used for the tuning of the instruments in liquid mode. No sample or blank was aspirated with the nebuliser during the LA analysis. The length of the LA transfer line was kept as short as possible to minimise the dead volume of the system. The temporal delay between a laser shot and the corresponding measurable ICP-MS signal was about 10-15 s. The LA system was equipped with a two-volume cell (called the TV2 cell in this design) to eliminate the cross-contamination of other samples and within the LA chamber. ${ }^{19}$ Detailed optimised instrumental settings and data acquisition parameters are reported in the ESI. $\uparrow$ The major $\mathrm{U}$ isotopes $\left({ }^{235} \mathrm{U}\right.$ and $\left.{ }^{238} \mathrm{U}\right)$ were measured on the Faraday detectors, while ion counters were used for the low abundant isotopes $\left({ }^{234} \mathrm{U}\right.$ and $\left.{ }^{236} \mathrm{U}\right)$. In order to minimise the tailing of ${ }^{235} \mathrm{U}$ on the $m / z=236$ peak, a retardation filter was used on the ion counter measuring ${ }^{236} \mathrm{U}$. This filter decreases the abundance sensitivity and separates ${ }^{235} \mathrm{U}^{+}$tailing from the ${ }^{236} \mathrm{U}^{+}$signal with a factor of $\sim 10$.

\section{Measurement of the U samples by LA-MC-ICP-MS}

The prepared sample pellets can be easily placed into the LA chamber. The raw data obtained by LA-MC-ICP-MS analysis were first corrected for the gas blank (Fig. 1A). The U isotope ratios were calculated subsequently as the ratios of the background-corrected signals after ablation. Data acquisition was performed only during the time interval when the ${ }^{235} \mathrm{U}$ ion beam intensity (the lowest signal on the Faraday detector) was exceeding $20 \mathrm{mV}$. Mass bias correction and ion counter gains were calculated using a NBS U-020 CRM pellet that was measured before the analysis of each solid sample. All results reported here are based on five replicate measurements of each sample. All uncertainties indicated are combined standard uncertainties, and they include the NBS U-020 and the sample measurement uncertainties, mass bias and ion counting gain 

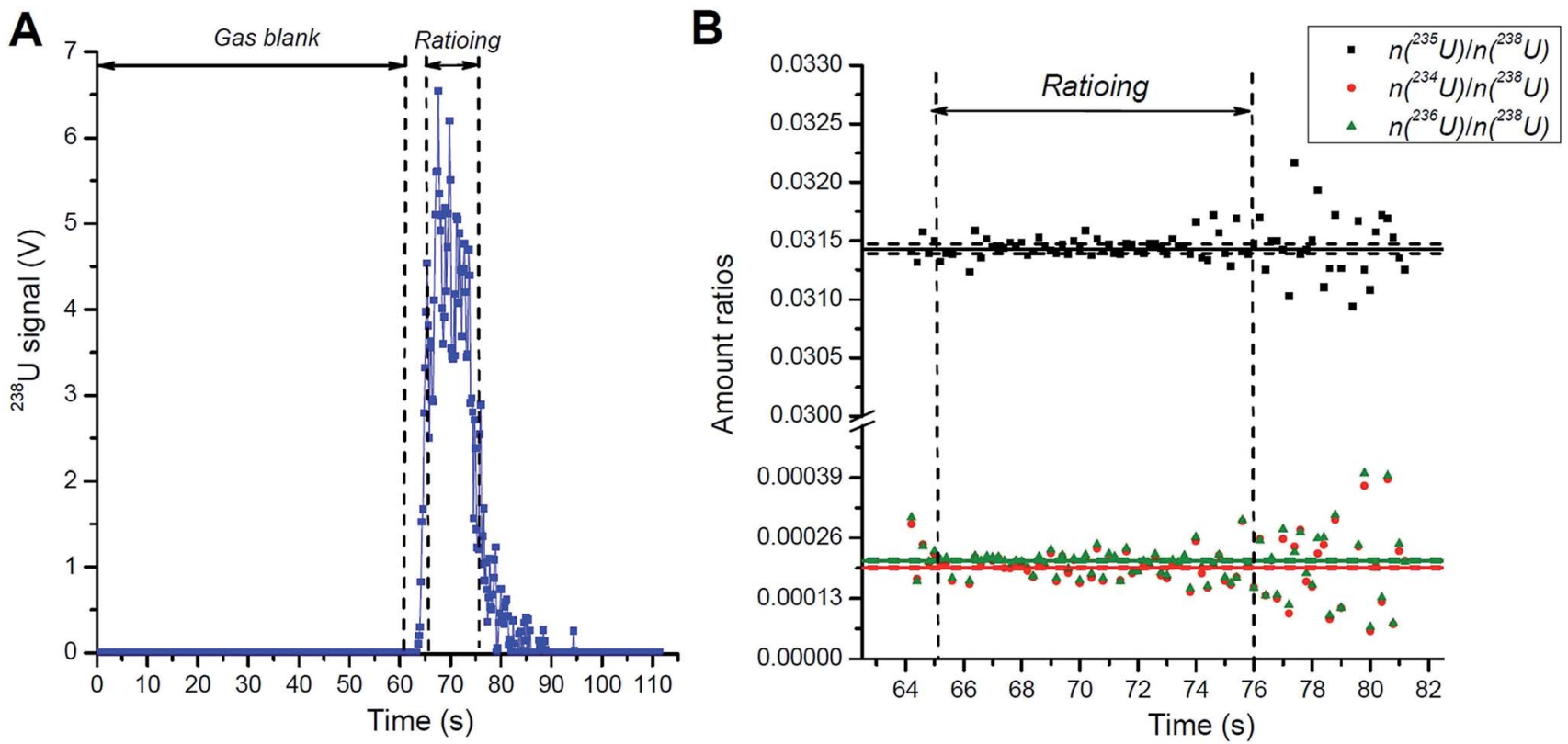

Fig. 1 (A) Laser ablation signal of ${ }^{238} \mathrm{U}$ indicating the time ranges for the data analysis and (B) measured $\mathrm{U}$ isotope ratios of the $\mathrm{U}-030$ sample. The certified ratios (solid line) and their respective uncertainties (dotted line) are shown in (B).

variations. They are given in parentheses and include a coverage factor of 2 ( $k=2$, approx. $95 \%$ confidence interval), and apply to the last two digits of the respective value.

\section{Investigations by scanning electron microscopy}

One sample was further investigated by scanning electron microscopy (SEM, Vega Tescan TS5130LSH, Czech Republic) after LA-MC-ICP-MS analysis. To this end, the sample was coated with a thin carbon layer to avoid charging effects on the surface aiming at producing clearer images. The SEM was operated at $20 \mathrm{kV}$ with a typical emission current of $50 \mu \mathrm{A}$.

\section{Results and discussion}

\section{Optimisation of measurement parameters}

The optimisation of the LA conditions (laser energy, ablation area and time, and repetition rate) was performed prior to the measurements. The main requirements were minimum material consumption and a maximum ICP-MS signal while avoiding the saturation of the detectors. Fig. 1A illustrates a typical ${ }^{238} \mathrm{U}$ signal acquired for the investigated U materials. As the obtained peak represents a transient signal, the length of the observed peak plateau increases with the duration of laser ablation. The applied fluence of the laser needs to be low and the ablation spot size has to be small, otherwise the detectors get saturated due to the high $\mathrm{U}$ signal. This limitation is caused by both the $\mathrm{U}$ matrix (high U content) and the employed TV2 LA cell. The cellin-a-cell design of the TV2 LA cell allows sampling of only a small portion of the entire gas volume of the LA cell. Therefore the ablated material is diluted in only a small volume, leading to elevated ICP-MS signals. Based on the experimental data acquired during this study, the low laser energy applied for LA did not cause any measurable isotopic fractionation that was higher than the solution-based method. For data evaluation rationing of the respective background-corrected signals was applied. Only measurements where the ${ }^{235} \mathrm{U}$ signal was higher than $20 \mathrm{mV}$ were considered for the calculation of the U isotope ratio. This way, the obtained ${ }^{235} \mathrm{U}$ signal is significantly higher than the spectral background yielding improved counting statistics. In this case the corresponding ${ }^{238} \mathrm{U}$ signal for samples containing natural $\mathrm{U}$ is about $2.8 \mathrm{~V}$. The applied time segments for acquiring the ICP-MS signals of the spectral background and for a representative sample are shown in Fig. 1A.

A potentially elevated spectral background or possible crosscontamination from other samples within the LA cell can be detected easily during the first approx. $60 \mathrm{~s}$ of the time resolved ICP-MS signal, i.e. during the blank measurement. The low ICPMS signal following the laser pulse reveals that the ablated material has been removed entirely from the LA cell (Fig. 1). The wash-out of the sample in our experimental set-up takes less than $10 \mathrm{~s}$ after the initial ICP-MS signal arising from the sample has dropped to $20 \mathrm{mV}$ (end of data acquisition) and returned to background levels, and thus the potential memory effects are minimal.

\section{Measurement of uranium isotope ratios}

For repeated laser shots on a specific sample, individual positions for each ablation were used. A representative example of the obtained $\mathrm{U}$ isotope ratios for the U-030 reference material is shown in Fig. 1B together with the corresponding certified $U$ isotope ratios and their respective uncertainties. The observed elevated deviation of the experimental amount ratios from the certified values towards the end of the ablation (Fig. 1B) resulted from the decreasing intensity of the ICP-MS signal, i.e. the 
poorer signal-to-background ratio. The measured $\mathrm{U}$ isotope amount ratios for the pelletized CRMs are shown in Table 1. These experimental values are in good agreement with the corresponding certified values. The approximate relative uncertainty ranges from 0.1 to $1.4 \%$ for the ${ }^{235} \mathrm{U} /{ }^{238} \mathrm{U}$ ratio, while this value is $1.8-6.1 \%$ for the minor ${ }^{234} \mathrm{U} /{ }^{238} \mathrm{U}$ ratio. The low uncertainties of the $\mathrm{U}$ isotopic measurements that are comparable to the CRM uncertainty not only demonstrate the high precision of the ICP-MS measurement but also prove that the employed reference materials are homogeneous on a microscopic level.

These numbers are comparable to the performance of solution-based magnetic sector ICP-MS analysis with a singledetector, but the analytical procedure applied in this study is distinctly faster and requires by far less sample material. ${ }^{9}$ The abundance of ${ }^{236} \mathrm{U}$ could also be measured accurately in low enriched and depleted U samples (Table 1). In the two UOCs containing natural $U$ isotopic composition the expected ${ }^{236} \mathrm{U} /{ }^{238} \mathrm{U}$ ratio is less than $10^{-9}$. In this case, the actual ${ }^{236} \mathrm{U} /{ }^{238} \mathrm{U}$ ratio is below the detection limit of $2 \times 10^{-6}$ of our experimental set-up. This detection limit was calculated taking into account the gas blank, the ${ }^{235} \mathrm{U}$ enrichment and the ${ }^{235} \mathrm{U}^{1} \mathrm{H}^{+}$signal contribution due to the hydride formation.

\section{SEM measurement of the ablated sample}

In order to estimate the amount of material consumed by LA measurements, the crater of an ablated sample was examined by scanning electron microscopy (SEM). Fig. 2 shows the crater of a pressed pellet formed during LA. By measuring the

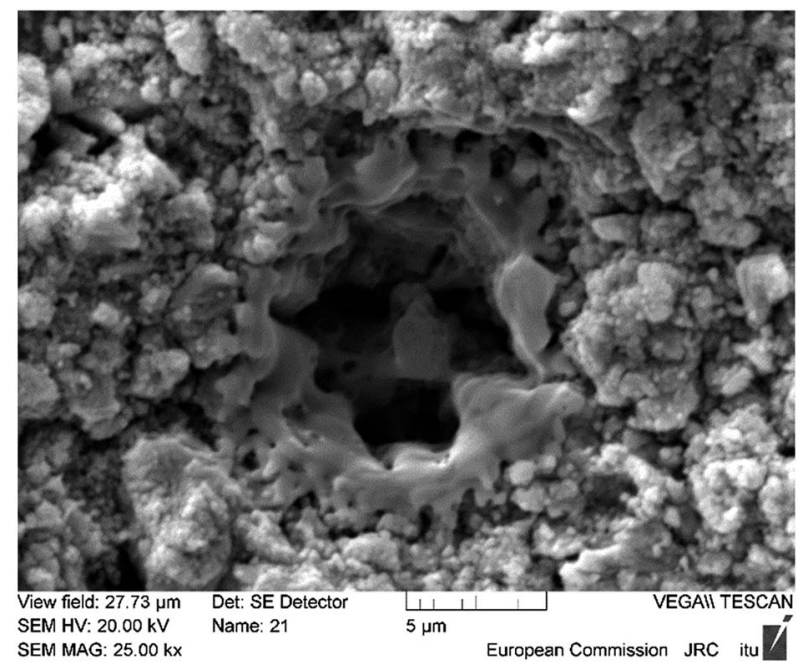

Fig. 2 Laser ablation crater analysed by SEM.

Table 1 Measured amount ratios of the investigated samples ( $n=5$ for each sample) together with the certified values. Uncertainties are given in parentheses and include a coverage factor of $k=2$. Relative uncertainties and the bias from the certified/reported value are also given

\begin{tabular}{|c|c|c|c|}
\hline Sample & ${ }^{235} \mathrm{U} /{ }^{238} \mathrm{U}$ & ${ }^{234} \mathrm{U} /{ }^{238} \mathrm{U}$ & ${ }^{236} \mathrm{U} /{ }^{238} \mathrm{U}$ \\
\hline \multicolumn{4}{|l|}{ U-005 } \\
\hline Measured & $0.004958(68) \pm 1.4 \%$ & $0.00002213(91) \pm 4.1 \%$ & $0.00004756(99) \pm 2.1 \%$ \\
\hline Certified & $0.004919(49) \pm 0.10 \%$ & $0.00002191(40) \pm 1.8 \%$ & $0.00004683(50) \pm 1.1 \%$ \\
\hline Bias & $0.78 \%$ & $1.0 \%$ & $1.6 \%$ \\
\hline \multicolumn{4}{|l|}{ U-010 } \\
\hline Measured & $0.010182(65) \pm 0.65 \%$ & $0.0000551(23) \pm 4.1 \%$ & $0.0000695(29) \pm 4.1 \%$ \\
\hline Certified & $0.010140(10) \pm 0.10 \%$ & $0.00005466(50) \pm 0.91 \%$ & $0.00006880(70) \pm 1.0 \%$ \\
\hline Bias & $0.41 \%$ & $0.74 \%$ & $1.1 \%$ \\
\hline \multicolumn{4}{|l|}{ U-015 } \\
\hline Measured & $0.015564(77) \pm 0.54 \%$ & $0.0000858(52) \pm 6.1 \%$ & $0.000166(11) \pm 6.6 \%$ \\
\hline Certified & $0.015565(16) \pm 0.10 \%$ & $0.0008634(91) \pm 1.1 \%$ & $0.0001666(10) \pm 0.60 \%$ \\
\hline Bias & $0.00 \%$ & $-0.59 \%$ & $-0.56 \%$ \\
\hline \multicolumn{4}{|l|}{ U-030 } \\
\hline Measured & $0.031428(94) \pm 0.30 \%$ & $0.0001980(36) \pm 1.8 \%$ & $0.0002116(38) \pm 1.8 \%$ \\
\hline Certified & $0.031430(31) \pm 0.10 \%$ & $0.0001960(10) \pm 0.51 \%$ & $0.0002105(10) \pm 0.48 \%$ \\
\hline Bias & $-0.01 \%$ & $1.0 \%$ & $0.76 \%$ \\
\hline \multicolumn{4}{|l|}{ IAEA-9064 } \\
\hline Measured & $0.0072534(56) \pm 0.10 \%$ & $0.00005474(97) \pm 1.8 \%$ & $<2 \times 10^{-6}$ \\
\hline Certified & $0.0072568(36) \pm 0.050 \%$ & $0.00005472(76) \pm 1.4 \%$ & $<2 \times 10^{-8}$ \\
\hline Bias & $-0.05 \%$ & $0.03 \%$ & \\
\hline \multicolumn{4}{|l|}{ IAEA-9449 } \\
\hline Measured & $0.007281(35) \pm 0.48 \%$ & $0.0000559(15) \pm 2.6 \%$ & $<2 \times 10^{-6}$ \\
\hline Certified $^{a}$ & $0.0072561(30) \pm 0.41 \%$ & $0.00005449(23) \pm 0.42 \%$ & $<1 \times 10^{-8}$ \\
\hline Bias & $0.34 \%$ & $2.5 \%$ & \\
\hline
\end{tabular}


diameter and the approximate depth of the produced crater and using the sample density of $8.3 \mathrm{~g} \mathrm{~cm}^{-3}$ for $\mathrm{U}_{3} \mathrm{O}_{8}$, the amount of material removed during LA was calculated. These calculations revealed that approximately $5 \mathrm{ng}$ of sample are consumed during a single LA-MC-ICP-MS measurement. The minute amount of material required for the analysis of the $\mathrm{U}$ isotopic composition using LA-MC-ICP-MS endorses the analytical procedure for nuclear forensic investigations.

\section{Conclusions}

A rapid method was developed and validated for the direct measurement of $U$ isotope ratios in solid nuclear samples. Due to the absence of any chemical sample preparation and an insignificant sample consumption of approx. $5 \mathrm{ng}$ of $\mathrm{U}$ per measurement, the analysed specimen remains macroscopically unaltered. The developed analytical method is particularly suited for the initial examination of nuclear materials, where different destructive techniques are subsequently applied. The $\mathrm{U}$ isotope ratios measured by LA-MC-ICP-MS show somewhat higher uncertainties than those measured by more time consuming methods such as TIMS or solutionbased MC-ICP-MS. However, when compared to other methods, such as gamma spectrometry, LA-MC-ICP-MS demonstrates distinctly superior performance. Moreover, using LA coupled to ICP-MS the sample dissolution can be avoided, largely reducing the amount of radioactive waste generated during analysis and essentially shortening the analysis time. All the above-mentioned aspects are fundamentally important for the investigative work performed in the area of nuclear forensics. As such LA-MC-ICP-MS is a powerful tool that can be applied favourably to the examination of seized nuclear samples.

The $\mathrm{U}$ isotope ratio measurements carried out in the current experiments were performed on homogeneous reference materials. The high spatial resolution (a few $\mu \mathrm{m}$ ) of LA-ICP-MS, however, also allows studying the inhomogeneity of the examined materials, as long as the area of inhomogeneity is comparable to the spot size of the applied laser beam. This capability will be tested for certified reference materials as well as for real world nuclear samples (such as surface U contamination or several $U$ enrichments within the same sample) in future studies. Moreover, LA-MC-ICP-MS will be used for measurements of impurities in nuclear specimens, for age dating of uranium materials employing the ${ }^{230} \mathrm{Th} /{ }^{234} \mathrm{U}$ ratio as well as for $\mathrm{U}$ isotope ratio measurements of radioactive particles.

\section{Conflicts of interest}

There are no conflicts to declare.

\section{References}

1 D. L. Donohue, Anal. Chem., 2002, 74, 28A-35A.

2 S. Boulyga, S. Konegger-Kappel, S. Richter and L. Sangély, J. Anal. At. Spectrom., 2015, 30, 1469-1489.

3 K. Mayer, M. Wallenius and Z. Varga, Chem. Rev., 2013, 113, 884-900.

4 M. J. Kristo, in Handbook of Radioactivity Analysis, 2013, pp. 1281-1304, DOI: 10.1016/B978-0-12-384873-4.00021-9.

5 K. Mayer, M. Wallenius and Z. Varga, Anal. Chem., 2015, 87, 11605-11610.

6 O. Pereira De Oliveira, W. De Bolle, S. Richter, A. Alonso, H. Kühn, J. E. S. Sarkis and R. Wellum, Int. J. Mass Spectrom., 2005, 246, 35-42.

7 L. Yang, Mass Spectrom. Rev., 2009, 28, 990-1011.

8 F. Albarède, P. Telouk, J. Blichert-Toft, M. Boyet, A. Agranier and B. Nelson, Geochim. Cosmochim. Acta, 2004, 68, 27252744.

9 D. C. Baxter, I. Rodushkin and E. Engström, J. Anal. At. Spectrom., 2012, 27, 1355-1381.

$10 \mathrm{~T}$. Kell and S. Jovanovic, Nuclear Forensics International Technical Working Group (ITWG), 22nd Annual Meeting, Karlsruhe, Germany, 28-30 June, 2017.

11 S. Kappel, S. F. Boulyga and T. Prohaska, J. Environ. Radioact., 2012, 113, 8-15.

12 N. S. Lloyd, R. R. Parrish, M. S. A. Horstwood and S. R. N. Chenery, J. Anal. At. Spectrom., 2009, 24, 752-758.

13 M. Guillong, P. Heimgartner, Z. Kopajtic, D. Günther and I. Günther-Leopold, J. Anal. At. Spectrom., 2007, 22, 399-402.

14 T. L. Spano, A. Simonetti, E. Balboni, C. Dorais and P. C. Burns, Appl. Geochem., 2017, 84, 277-285.

15 Z. Stefanka, R. Katona and Z. Varga, J. Anal. At. Spectrom., 2008, 23, 1030-1033.

16 R. C. Marin, J. E. S. Sarkis and M. R. L. Nascimento, J. Radioanal. Nucl. Chem., 2013, 295, 99-104.

17 S. Bürger, S. F. Boulyga, M. V. Peńkin, D. Bostick, S. Jovanovic, R. Lindvall, G. Rasmussen and L. Riciputi, J. Radioanal. Nucl. Chem., 2014, 301, 711-729.

18 Standard Reference Materials: Uranium Isotopic Standard Reference Materials, ed. E. L. Garner, L. A. Machlan and W. R. Shields, National Bureau of Standards Special Publication 260-27, USA, 1971.

19 W. Müller, M. Shelley, P. Miller and S. Broude, J. Anal. At. Spectrom., 2009, 24, 209-214. 